\title{
EXACT TRAVELING-WAVE SOLUTION FOR LOCAL FRACTIONAL BOUSSINESQ EQUATION IN FRACTAL DOMAIN
}

\author{
XIAO-JUN YANG, ${ }^{*}$ 『 J. A. TENREIRO MACHADO ${ }^{\dagger}$ \\ and DUMITRU BALEANU $\$, \S$ \\ *State Key Laboratory for Geo-Mechanics and Deep Underground Engineering \\ School of Mechanics and Civil Engineering \\ China University of Mining and Technology \\ Xuzhou 221116, P. R. China \\ ${ }^{\dagger}$ Institute of Engineering, Polytechnic of Porto \\ Department of Electrical Engineering \\ Rua Dr. António Bernardino de Almeida, 4249-015 Porto, Portugal \\ ${ }^{\ddagger}$ Department of Mathematics \\ Cankya University, Ogretmenler Cad. 14, Balgat-06530, Ankara, Turkey \\ $\S$ Institute of Space Sciences, Magurele-Bucharest, Romania \\ 『dyangxiaojun@163.com
}

Received February 23, 2017

Revised March 29, 2017

Accepted April 6, 2017

Published June 2, 2017

\begin{abstract}
The new Boussinesq-type model in a fractal domain is derived based on the formulation of the local fractional derivative. The novel traveling wave transform of the non-differentiable type is adopted to convert the local fractional Boussinesq equation into a nonlinear local fractional ODE. The exact traveling wave solution is also obtained with aid of the non-differentiable
\end{abstract}

\footnotetext{
『Corresponding author.
}

This is an Open Access article published by World Scientific Publishing Company. It is distributed under the terms of the Creative Commons Attribution 4.0 (CC-BY) License. Further distribution of this work is permitted, provided the original work is properly cited. 
graph. The proposed method, involving the fractal special functions, is efficient for finding the exact solutions of the nonlinear PDEs in fractal domains.

Keywords: Exact Traveling-Wave Solution; Local Fractional Boussinesq Equation; Local Fractional Derivative; Fractals.

\section{INTRODUCTION}

Fractional-order derivatives (FDs) have successfully been applied for describing fractal problems in engineering 17 Recent examples are the heat transport in fractal media, ${ }^{9}$ fractal hydrodynamic equations, ${ }^{[0]}$ fractal electrostatics ${ }^{[11}$ fractal FokkerPlanck equations 12 and fractal description of stress and strain in elasticity 13

There are several alternative approaches for describing the complex and fractal behaviors in nature 1 - 8 The theory of the local fractional derivative (LFD) is a mathematical tool for describing fractals, that was used to model the fractal complexity in shallow water surfaces, $\frac{14}{14}$ LC-electric circuit, 15 traveling-wave solution of the Burgerstype equation, 16 PDEs, $17 \sqrt[20]{20}$ ODEs, 21 and inequalities.22123 The useful models for the LFD were considered $^{24} \sqrt{29}$ and discussed. $\stackrel{30}{ }$ However, the nonlinear local fractional Boussinesq equations and their non-differentiable-type traveling-wave solutions have not yet been tackled. The main aim of the paper is to derive the Boussinesq-type model in fractal domain and to find the exact nondifferentiable-type traveling-wave solution for the two-dimensional problem.

The structure of the article is as follows. In Sec. 2, the theory of the LFD is presented. In Sec. 3, the local fractional Boussinesq equation for the wave content in fractal domain is derived. In Secs. 4 and 5. the traveling-wave transform and the exact solutions are discussed, respectively. Finally, the conclusions are drawn in Sec. 6.

\section{PRELIMINARIES}

In this section, the concept and properties of the LFD are introduced. The fractal special functions (FSFs) defined on fractal sets for the fractaldimensional parameters from 1 to $\ln 2 / \ln 3$ are also given. Let $\mathrm{C}_{\delta}(r, s)$ be a set of the local fractional continuous functions (LFCFs) with the fractal dimension $\delta$ such that $0<\delta<1$. For more details of the LFCFs, see Refs. 1, 14, 15, 16, 23.

Definition 1. Let $M_{\delta}(\tau) \in \mathrm{C}_{\delta}(r, s)$. The LFD of $M_{\delta}(\tau)$ of fractal order $\delta(0<\delta<1)$ at the point $\tau=\tau_{0}$ is given as 1114 16]23:

$$
\begin{aligned}
D^{(\delta)} M_{\delta}\left(\tau_{0}\right) & =\frac{d^{\delta} M_{\delta}\left(\tau_{0}\right)}{d \tau^{\delta}} \\
& =\lim _{\tau \rightarrow \tau_{0}} \frac{\Delta^{\delta}\left(M_{\delta}(\tau)-M_{\delta}\left(\tau_{0}\right)\right)}{\left(\tau-\tau_{0}\right)^{\delta}},
\end{aligned}
$$

where

$$
\begin{aligned}
& \Delta^{\delta}\left(M_{\delta}(\tau)-M_{\delta}\left(\tau_{0}\right)\right) \\
& \quad \cong \Gamma(1+\delta) \Delta\left[M_{\delta}(\tau)-M_{\delta}\left(\tau_{0}\right)\right] .
\end{aligned}
$$

Definition 2. The local fractional partial derivative (LFPD) of the function $M_{\delta}(\mu, \tau)$ of fractal order $\delta(0<\delta<1)$ at the point $\tau=\tau_{0}$ is defined as 1 :

$$
\frac{\partial^{\delta} M_{\delta}\left(\mu, \tau_{0}\right)}{\partial \tau^{\delta}}=\lim _{\tau \rightarrow \tau_{0}} \frac{\Delta^{\delta}\left(M_{\delta}(\mu, \tau)-M_{\delta}\left(\mu, \tau_{0}\right)\right)}{\left(\tau-\tau_{0}\right)^{\delta}},
$$

where

$$
\begin{aligned}
& \Delta^{\delta}\left(M_{\delta}(\mu, \tau)-M_{\delta}\left(\mu, \tau_{0}\right)\right) \\
& \quad \cong \Gamma(1+\delta) \Delta\left[M_{\delta}(\mu, \tau)-M_{\delta}\left(\mu, \tau_{0}\right)\right] .
\end{aligned}
$$

The LFPD of the function $M_{\delta}(\mu, \tau)$ of fractal order $\kappa \delta$ at the point $\tau=\tau_{0}$ is given as 1 :

$$
\frac{\partial^{\kappa \delta} M_{\delta}\left(\mu, \tau_{0}\right)}{\partial \tau^{\kappa \delta}}=\overbrace{\frac{\partial^{\delta}}{\partial \tau^{\delta}} \cdots \frac{\partial^{\delta}}{\partial \tau^{\delta}}}^{\kappa \text {-times }} M_{\delta}\left(\mu, \tau_{0}\right),
$$

where $0<\delta<1, \kappa \in N_{0}$ and $N_{0}$ is the set of integer numbers.

If $D^{(\delta)} M_{\delta, 1}(\tau)$ and $D^{(\delta)} M_{\delta, 2}(\tau)$ exist, then the operations of the LFCFs $M_{\delta, 1}(\tau)$ and $M_{\delta, 2}(\tau)$ are given as follows $1 \mid 14$.

$$
\begin{aligned}
D^{(\delta)} & {\left[M_{\delta, 1}(\tau) \pm M_{\delta, 2}(\tau)\right] } \\
& =D^{(\delta)} M_{\delta, 1}(\tau) \pm D^{(\delta)} M_{\delta, 2}(\tau),
\end{aligned}
$$

$$
\begin{aligned}
D^{(\delta)}[ & \left.M_{\delta, 1}(\tau) M_{\delta, 2}(\tau)\right] \\
= & {\left[D^{(\delta)} M_{\delta, 1}(\tau)\right] M_{\delta, 2}(\tau) } \\
& +M_{\delta, 1}(\tau)\left[D^{(\delta)} M_{\delta, 2}(\tau)\right],
\end{aligned}
$$


Table 1 The Expressions of the FSFs.

\begin{tabular}{lc}
\hline FSFs & Expressions \\
\hline $\mathrm{O}_{\delta}\left(\tau^{\delta}\right)$ & $\sum_{\kappa=0}^{\infty} \tau^{\kappa \delta} / \Gamma(1+\kappa \delta)$ \\
$\mathrm{O}_{\delta}\left(\theta \tau^{\delta}\right)$ & $\sum_{\kappa=0}^{\infty} \theta^{\kappa} \tau^{\kappa \delta} / \Gamma(1+\kappa \delta)$ \\
\hline
\end{tabular}

Table 2 The LFDs of the FSFs Defined on Fractal Sets.

\begin{tabular}{lc}
\hline FSFs & LFDs \\
\hline $\mathrm{O}_{\delta}\left(\tau^{\delta}\right)$ & $D^{(\delta)} \mathrm{O}_{\delta}\left(\tau^{\delta}\right)=\mathrm{O}_{\delta}\left(\tau^{\delta}\right)$ \\
$\mathrm{O}_{\delta}\left(\rho \tau^{\delta}\right)$ & $D^{(\delta)} \mathrm{O}_{\delta}\left(\rho \tau^{\delta}\right)=\rho \mathrm{O}_{\delta}\left(\rho \tau^{\delta}\right)$ \\
\hline
\end{tabular}

$(M 3)$

$$
\begin{aligned}
D^{(\delta)}\left[M_{\delta, 1}(\tau) / M_{\delta, 2}(\tau)\right] \\
=\left\{\left[D^{(\delta)} M_{\delta, 1}(\tau)\right] M_{\delta, 2}(\tau)\right. \\
\left.\quad-M_{\delta, 1}(\tau)\left[D^{(\delta)} M_{\delta, 2}(\tau)\right]\right\} / M_{\delta, 2}^{2},
\end{aligned}
$$

provided that $M_{\delta, 2}(\tau) \neq 0$.

If $\theta$ is a constant and $\kappa \in N_{0}$, then the expressions of the FSFs defined on fractal sets $1114 ! 16$ are listed in Table 1

If $\rho$ is a constant, then the LFDs of the FSFs defined on fractal sets ${ }^{1}$ are listed in Table 2 .

\section{THE BOUSSINESQ-TYPE MODEL IN FRACTAL DOMAIN}

In this section, from the theory of LFD view of point, we derive the two-dimensional and threedimensional local fractional Boussinesq equations in fractal domain.

The local fractional PDEs of the threedimensional free surface for the fractal incompresssible fluid on the flat bottom are described as:

$$
\begin{gathered}
\frac{\partial^{2 \delta} M_{\delta}}{\partial \mu^{2 \delta}}+\frac{\partial^{2 \delta} M_{\delta}}{\partial \xi^{2 \delta}}+\frac{\partial^{2 \delta} M_{\delta}}{\partial \omega^{2 \delta}}=0, \\
\frac{\partial^{\delta} \Lambda_{\delta}}{\partial \tau^{\delta}}+\frac{\partial^{\delta} M_{\delta}}{\partial \mu^{\delta}} \frac{\partial^{\delta} \Lambda_{\delta}}{\partial \mu^{\delta}}+\frac{\partial^{\delta} M_{\delta}}{\partial \xi^{\delta}} \frac{\partial^{\delta} \Lambda_{\delta}}{\partial \xi^{\delta}} \\
-\frac{\partial^{\delta} M_{\delta}}{\partial \omega^{\delta}}=0, \quad \omega=H_{\delta},
\end{gathered}
$$

$$
\begin{aligned}
\frac{\partial^{\delta} \Lambda_{\delta}}{\partial \tau^{\delta}}+\frac{1}{2}\left(\left(\frac{\partial^{\delta} \Lambda_{\delta}}{\partial \mu^{\delta}}\right)^{2}+\left(\frac{\partial^{\delta} \Lambda_{\delta}}{\partial \xi^{\delta}}\right)^{2}+\left(\frac{\partial^{\delta} \Lambda_{\delta}}{\partial \omega^{\delta}}\right)^{2}\right) \\
+\gamma H_{\delta}=0, \quad \omega=H_{\delta} \\
\frac{\partial^{\delta} \Lambda_{\delta}}{\partial \omega^{\delta}}=0, \quad \omega=0
\end{aligned}
$$

where

$$
H_{\delta}=H_{\delta, 0}+M_{\delta}(\mu, \xi, \tau)
$$

represents the local depth with the average depth $H_{\delta, 0}, \gamma$ is the gravitational constant, $M_{\delta}=M_{\delta}(\mu$, $\xi, \omega, \tau)$ and $\omega$ is the distance from the bottom.

The expression

$$
\begin{aligned}
\psi(\widetilde{\mu, \xi, \omega, \tau)=} & \frac{\partial^{\delta} \Lambda_{\delta}(\mu, \xi, \omega, \tau)}{\partial \mu^{\delta}} \widehat{i^{\delta}}+\frac{\partial^{\delta} \Lambda_{\delta}(\mu, \xi, \omega, \tau)}{\partial \xi^{\delta}} \widehat{j^{\delta}} \\
& +\frac{\partial^{\delta} \Lambda_{\delta}(\mu, \xi, \omega, \tau)}{\partial \omega^{\delta}} \widehat{k^{\delta}}
\end{aligned}
$$

describes the fractal fluid velocity using the quaternionic number system in fractal space $\widehat{i^{\delta}}, \widehat{j^{\delta}}$ and $\widehat{k^{\delta}}$ (see Ref. 1).

We have

$$
\begin{gathered}
\frac{\partial^{\delta} H_{\delta}}{\partial \tau^{\delta}}+\frac{\partial^{\delta}}{\partial \mu^{\delta}}\left(H_{\delta} \frac{\partial^{\delta} M_{\delta}}{\partial \mu^{\delta}}\right)+\frac{\partial^{\delta}}{\partial \xi^{\delta}}\left(H_{\delta} \frac{\partial^{\delta} M_{\delta}}{\partial \xi^{\delta}}\right)=0 \\
\frac{\partial^{\delta} \Lambda_{\delta}}{\partial \mu^{\delta}}=\frac{\partial^{\delta} M_{\delta}}{\partial \xi^{\delta}}
\end{gathered}
$$

where $H_{\delta}=H_{\delta, 0}+M_{\delta}(\mu, \xi, \tau), M_{\delta}=M_{\delta}(\mu, \xi, \omega, \tau)$ and $\Lambda_{\delta}=\Lambda_{\delta}(\mu, \xi, \omega, \tau)$.

The local fractional PDE of the fractal wave content in the three-dimensional case is

$$
\frac{\partial^{\delta} M_{\delta}}{\partial \tau^{\delta}}+\varsigma_{1} M_{\delta} \frac{\partial^{\delta} M_{\delta}}{\partial \mu^{\delta}}+\varsigma_{2} \frac{\partial^{3 \delta} M_{\delta}}{\partial \mu^{3 \delta}}+\sigma \frac{\partial^{\delta} \Lambda_{\delta}}{\partial \xi^{\delta}}=0,
$$

where $\sigma, \varsigma_{1}$ and $\varsigma_{2} \in R^{+}$are parameters.

Substituting Eq. (9) into Eq. (10), we have the three-dimensional local fractional Boussinesq equation for the wave content in the fractal domain:

$$
\begin{aligned}
& \frac{\partial^{\delta}}{\partial \mu^{\delta}}\left(\frac{\partial^{\delta} M_{\delta}}{\partial \tau^{\delta}}+\varsigma_{1} M_{\delta} \frac{\partial^{\delta} M_{\delta}}{\partial \mu^{\delta}}+\varsigma_{2} \frac{\partial^{3 \delta} M_{\delta}}{\partial \mu^{3 \delta}}\right) \\
& \quad+\sigma \frac{\partial^{2 \delta} M_{\delta}}{\partial \xi^{2 \delta}}=0 .
\end{aligned}
$$

The local fractional PDE in the one-dimensional fractal space can be written as

$$
\frac{\partial^{2 \delta} M_{\delta}}{\partial \tau^{2 \delta}}=\sigma^{2} \frac{\partial^{2 \delta} \Xi_{\delta}}{\partial \mu^{2 \delta}}
$$


which leads to

$$
\begin{aligned}
& \frac{\partial^{\delta} M_{\delta}}{\partial \tau^{\delta}}=\sigma \frac{\partial^{\delta} \Xi_{\delta}}{\partial \mu^{\delta}} \\
& \frac{\partial^{\delta} \Xi_{\delta}}{\partial \tau^{\delta}}=\sigma \frac{\partial^{\delta} M_{\delta}}{\partial \mu^{\delta}},
\end{aligned}
$$

where $\Xi_{\delta}=\Xi_{\delta}(\mu, \tau)$ and $M_{\delta}=M_{\delta}(\mu, \tau)$ are the non-differentiable functions and $\sigma(\sigma>0)$ is an unknown constant.

When $M_{\delta}(\mu, \tau)=\Xi_{\delta}(\mu, \tau)$, Eq. (16) can be written as:

$$
\frac{\partial^{2 \delta} M_{\delta}}{\partial \tau^{2 \delta}}=\sigma^{2} \frac{\partial^{2 \delta} M_{\delta}}{\partial \mu^{2 \delta}},
$$

which is the local fractional wave equation in the one-dimensional fractal space 1

When $M_{\delta}(\mu, \tau)=\Xi_{\delta}(\mu, \tau)$ in Eq. (17), the local fractional conservation equation for the onedimensional fractal waves is given as ${ }^{1}$ :

$$
\frac{\partial^{\delta} M_{\delta}}{\partial \tau^{\delta}}=\sigma \frac{\partial^{\delta} M_{\delta}}{\partial \mu^{\delta}},
$$

where $M_{\delta}=M_{\delta}(\mu, \tau)$.

Taking

$$
\frac{\partial^{\delta} \Lambda_{\delta}}{\partial \xi^{\delta}}=\frac{\partial^{\delta} M_{\delta}}{\partial \mu^{\delta}}
$$

in Eq. (14), we have the local fractional PDE for a fractal velocity potential

$$
\frac{\partial^{\delta} M_{\delta}}{\partial \tau^{\delta}}=\sigma \frac{\partial^{\delta} M_{\delta}}{\partial \mu^{\delta}}
$$

such that

$$
\frac{\partial^{\delta} M_{\delta}}{\partial \tau^{\delta}}+\varsigma_{1} M_{\delta} \frac{\partial^{\delta} M_{\delta}}{\partial \mu^{\delta}}+\varsigma_{2} \frac{\partial^{3 \delta} M_{\delta}}{\partial \mu^{3 \delta}}+\sigma \frac{\partial^{\delta} M_{\delta}}{\partial \mu^{\delta}}=0,
$$

where $\varsigma_{1}$ and $\varsigma_{2}$ are two parameters. Equation (6) represents the local fractional PDE for the fractal wave content in the two-dimensional case.

For $\sigma=1$, we obtain from Eq. (6) that

$$
\frac{\partial^{\delta} M_{\delta}}{\partial \tau^{\delta}}+\varsigma_{1} M_{\delta} \frac{\partial^{\delta} M_{\delta}}{\partial \mu^{\delta}}+\varsigma_{2} \frac{\partial^{3 \delta} M_{\delta}}{\partial \mu^{3 \delta}}+\frac{\partial^{\delta} M_{\delta}}{\partial \mu^{\delta}}=0 .
$$

Taking $\sigma=0$ in Eq. (6), we obtain the local fractional Korteweg-de Vries equation ${ }^{114}$

$$
\frac{\partial^{\delta} M_{\delta}}{\partial \tau^{\delta}}+\varsigma_{1} M_{\delta} \frac{\partial^{\delta} M_{\delta}}{\partial \mu^{\delta}}+\varsigma_{2} \frac{\partial^{3 \delta} M_{\delta}}{\partial \mu^{3 \delta}}=0 .
$$

With a similar procedure, we have the local fractional PDE of a fractal velocity potential in the twodimensional case given by

$$
\frac{\partial^{\delta} M_{\delta}}{\partial \mu^{\delta}}=\frac{\partial^{\delta} M_{\delta}}{\partial \xi^{\delta}}
$$

such that

$$
\frac{\partial^{\delta} M_{\delta}}{\partial \tau^{\delta}}+\varsigma_{1} M_{\delta} \frac{\partial^{\delta} M_{\delta}}{\partial \mu^{\delta}}+\varsigma_{2} \frac{\partial^{3 \delta} M_{\delta}}{\partial \mu^{3 \delta}}+\sigma \frac{\partial^{\delta} M_{\delta}}{\partial \xi^{\delta}}=0 .
$$

Substituting Eq. (26) into Eq. (27), we have

$$
\begin{aligned}
& \frac{\partial^{\delta}}{\partial \mu^{\delta}}\left(\frac{\partial^{\delta} M_{\delta}}{\partial \tau^{\delta}}+\varsigma_{1} M_{\delta} \frac{\partial^{\delta} M_{\delta}}{\partial \mu^{\delta}}+\varsigma_{2} \frac{\partial^{3 \delta} M_{\delta}}{\partial \mu^{3 \delta}}\right) \\
& \quad+\sigma \frac{\partial^{2 \delta} M_{\delta}}{\partial \mu^{2 \delta}}=0
\end{aligned}
$$

where $\sigma, \varsigma_{1}$ and $\varsigma_{2}$ are the parameters and $M_{\delta}=$ $M_{\delta}(\mu, \tau)$.

Equation (28) is the two-dimensional local fractional Boussinesq equation for the wave content in the fractal domain.

\section{TRAVELING-WAVE TRANSFORM TECHNOLOGY}

In this section, the traveling-wave transformation technology for finding the exact solution for the nonlinear PDEs is considered.

We consider the following nonlinear local fractional PDE:

$$
\begin{aligned}
& \Theta_{\delta}\left(\frac{\partial^{2 \delta} M_{\delta}(\mu, \tau)}{\partial \mu^{2 \delta}}, \ldots, \frac{\partial^{3 \delta} M_{\delta}(\mu, \tau)}{\partial \mu^{3 \delta}}, \frac{\partial^{\delta} M_{\delta}(\mu, \tau)}{\partial t^{\delta}}\right) \\
& \quad=0
\end{aligned}
$$

where $\Theta_{\delta}=\Theta_{\delta}(\mu, \tau)$ is the nonlinear local fractional operator. 1

The non-differentiable traveling-wave transformation is defined by

$$
\psi^{\delta}=\mu^{\delta}-\nu^{\delta} \tau^{\delta},
$$

where

$$
\lim _{\delta \rightarrow 1} \psi=\mu-\nu \tau .
$$

With the aid of Eqs. (30) and (31), we consider

$$
\Theta_{\delta}(\mu, \tau)=\Theta_{\delta}(\psi)
$$


Following the chain rule of the LFD, we have from Eq. (30) that

$$
\begin{aligned}
\frac{\partial^{\delta} \Theta_{\delta}(\mu, \tau)}{\partial \tau^{\delta}} & =\frac{\partial^{\delta} \Theta_{\delta}(\mu, \tau)}{\partial \psi^{\delta}}\left(\frac{\partial \psi}{\partial \tau}\right)^{\delta} \\
& =-\nu^{\delta} \frac{\partial^{\delta} \Theta_{\delta}(\psi)}{\partial \psi^{\delta}} \\
\frac{\partial^{\delta} \Theta_{\delta}(\mu, \tau)}{\partial \mu^{\delta}} & =\frac{\partial^{\delta} \Theta_{\delta}(\psi)}{\partial \psi^{\delta}} \\
\frac{\partial^{2 \delta} \Theta_{\delta}(\mu, \tau)}{\partial \mu^{2 \delta}} & =\frac{\partial^{2 \delta} \Theta_{\delta}(\psi)}{\partial \psi^{2 \delta}} \\
\frac{\partial^{3 \delta} \Theta_{\delta}(\mu, \tau)}{\partial \mu^{3 \delta}} & =\frac{\partial^{3 \delta} \Theta_{\delta}(\psi)}{\partial \psi^{3 \delta}} .
\end{aligned}
$$

Thus, making use of Eqs. (33)-(35), Eq. (29) can be rewritten as:

$$
\Theta_{\delta}\left(\frac{d^{2 \delta} \Theta_{\delta}(\psi)}{d \psi^{2 \delta}}, \ldots, \frac{d^{3 \delta} \Theta_{\delta}(\psi)}{d \psi^{3 \delta}}, \frac{d^{\delta} \Theta_{\delta}(\psi)}{d \psi^{\delta}}\right)=0,
$$

where $d^{3 \delta} \Theta_{\delta}(\psi) / d \psi^{3 \delta}, \quad d^{2 \delta} \Theta_{\delta}(\psi) / d \psi^{2 \delta}$ and $d^{\delta} \Theta_{\delta}$ $(\psi) / d \psi^{\delta}$ are the LFDs of the orders $3 \delta, 2 \delta$ and $\delta$ with respect to $\psi$, respectively.

We obtain the exact solutions of the nonlinear ODE for Eq. (37). With the help of Eq. (30), the exact traveling-wave solutions of Eq. (29) is also given.

\section{EXACT TRAVELING-WAVE SOLUTION FOR BOUSSINESQ-TYPE MODEL IN FRACTAL DOMAIN}

In this section, we find the exact traveling-wave solution for the local fractional local fractional Boussinesq equation.

Finding the local fractional integral of Eq. (28) with respect to $\mu$ yields

$$
\begin{gathered}
\frac{\partial^{\delta} M_{\delta}}{\partial \tau^{\delta}}+\varsigma_{1} M_{\delta} \frac{\partial^{\delta} M_{\delta}}{\partial \mu^{\delta}}+\varsigma_{2} \frac{\partial^{3 \delta} M_{\delta}}{\partial \mu^{3 \delta}}+\sigma \frac{\partial^{\delta} M_{\delta}}{\partial \mu^{\delta}} \\
=\Phi_{\delta}(\tau),
\end{gathered}
$$

where $\Phi_{\delta}(\tau)$ is the unknown constant.

Making $\Phi_{\delta}(\tau)=0$, Eq. (38) becomes

$$
\frac{\partial^{\delta} M_{\delta}}{\partial \tau^{\delta}}+\varsigma_{1} M_{\delta} \frac{\partial^{\delta} M_{\delta}}{\partial \mu^{\delta}}+\varsigma_{2} \frac{\partial^{3 \delta} M_{\delta}}{\partial \mu^{3 \delta}}+\sigma \frac{\partial^{\delta} M_{\delta}}{\partial \mu^{\delta}}=0 .
$$

Substituting Eqs. (33), (34) and (36) into Eq. (28) leads to the following nonlinear local fractional
ODE:

$\varsigma_{1} \Theta_{\delta}(\psi) \frac{d^{\delta} \Theta_{\delta}(\psi)}{d \psi^{\delta}}+\varsigma_{2} \frac{d^{3 \delta} \Theta_{\delta}(\psi)}{d \psi^{3 \delta}}+\varsigma_{3} \frac{d^{\delta} \Theta_{\delta}(\psi)}{d \psi^{\delta}}=0$,

where $M_{\delta}(\mu, \tau)=M_{\delta}(\psi)=\Theta_{\delta}(\mu, \tau)=\Theta_{\delta}(\psi)$ and $\varsigma_{3}=\sigma-\nu^{\delta}$.

Following the chain rule of the LFD, Eq. (40) is

$$
\frac{d^{\delta}}{d \psi^{\delta}}\left(\varsigma_{2} \frac{d^{2 \delta} \Theta_{\delta}(\psi)}{d \psi^{2 \delta}}+\varsigma_{3} \Theta_{\delta}(\psi)+\frac{\varsigma_{1}}{2} \Theta_{\delta}^{2}(\psi)\right)=0 .
$$

Finding the local fractional integral of Eq. (41) with respect to $\psi$ yields

$$
\varsigma_{2} \frac{d^{2 \delta} \Theta_{\delta}(\psi)}{d \psi^{2 \delta}}+\varsigma_{3} \Theta_{\delta}(\psi)+\frac{\varsigma_{1}}{2} \Theta_{\delta}^{2}(\psi)=\alpha_{1},
$$

where $\alpha_{1}$ is a constant.

Taking $\alpha_{1}=0$, we have from Eq. (42) that

$$
\frac{d^{2 \delta} \Theta_{\delta}(\psi)}{d \psi^{2 \delta}}+\frac{\varsigma_{3}}{\varsigma_{2}} \Theta_{\delta}(\psi)+\frac{\varsigma_{1}}{2 \varsigma_{2}} \Theta_{\delta}^{2}(\psi)=0 .
$$

Multiplying Eq. (19) by the term $2 \frac{d^{\delta} \Theta_{\delta}(\psi)}{d \psi^{\delta}}$, it follows that

$$
\begin{gathered}
2 \frac{d^{\delta} \Theta_{\delta}(\psi)}{d \psi^{\delta}} \frac{d^{2 \delta} \Theta_{\delta}(\psi)}{d \psi^{2 \delta}}+\frac{2 \varsigma_{3}}{\varsigma_{2}} \frac{d^{\delta} \Theta_{\delta}(\psi)}{d \psi^{\delta}} \Theta_{\delta}(\psi) \\
+\frac{\varsigma_{1}}{\varsigma_{2}} \frac{d^{\delta} \Theta_{\delta}(\psi)}{d \psi^{\delta}} \Theta_{\delta}^{2}(\psi)=0 .
\end{gathered}
$$

From Eq. (44), we have

$$
\begin{aligned}
\frac{d^{\delta}}{d \psi^{\delta}} & {\left[\left(\frac{d^{\delta} \Theta_{\delta}(\psi)}{d \psi^{\delta}}\right)^{2}+\left(\frac{\varsigma_{3}}{\varsigma_{2}} \Theta_{\delta}^{2}(\psi)\right)+\left(\frac{\varsigma_{1}}{3 \varsigma_{2}} \Theta_{\delta}^{3}(\psi)\right)\right] } \\
& =0,
\end{aligned}
$$

which, by finding the local fractional integral of Eq. (45), leads to

$$
\left(\frac{d^{\delta} \Theta_{\delta}(\psi)}{d \psi^{\delta}}\right)^{2}+\frac{\varsigma_{3}}{\varsigma_{2}} \Theta_{\delta}^{2}(\psi)+\frac{\varsigma_{1}}{3 \varsigma_{2}} \Theta_{\delta}^{3}(\psi)=\alpha_{2},
$$

where $\alpha_{2}$ is a constant.

Taking $\alpha_{2}=0$, we obtain from Eq. (46) that

$$
\left(\frac{d^{\delta} \Theta_{\delta}(\psi)}{d \psi^{\delta}}\right)^{2}+\frac{\varsigma_{3}}{\varsigma_{2}} \Theta_{\delta}^{2}(\psi)+\frac{\varsigma_{1}}{3 \varsigma_{2}} \Theta_{\delta}^{3}(\psi)=0 .
$$




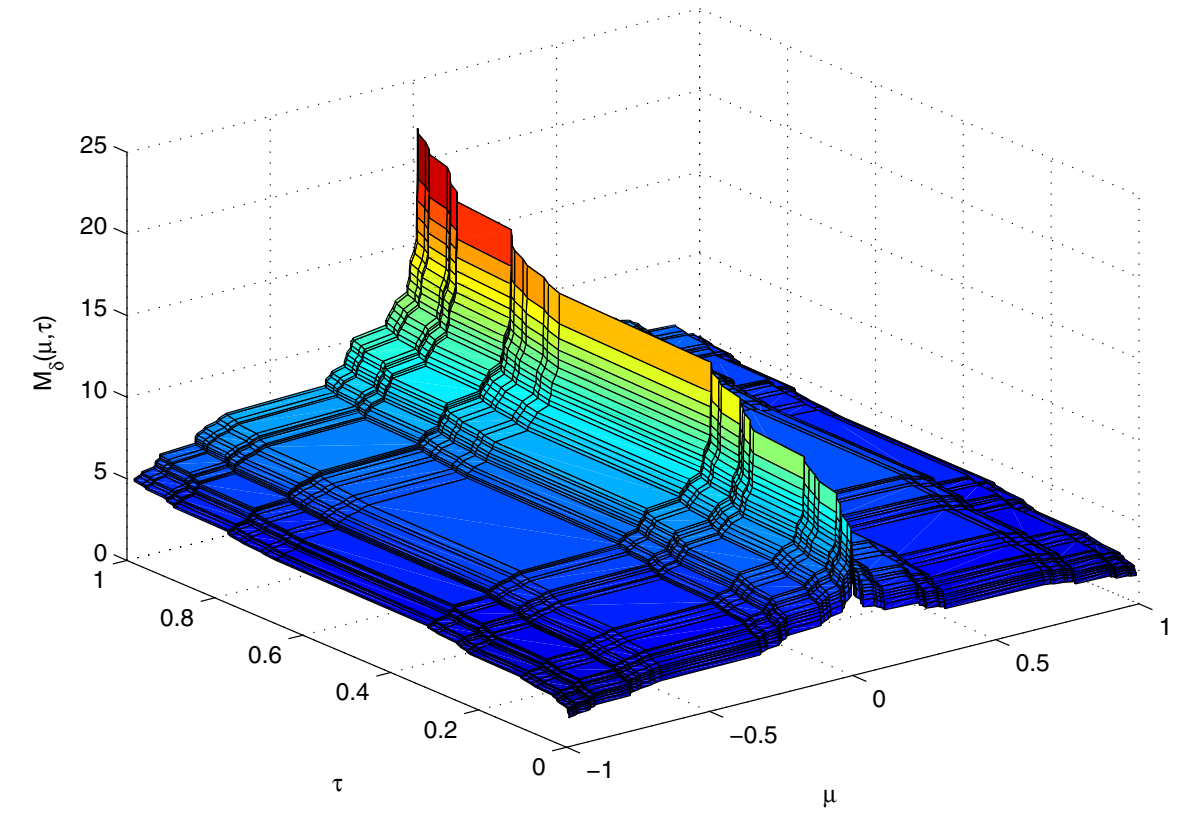

Fig. 1 The exact travelling-wave solution for the local fractional local fractional Boussinesq equation for the parameters $\sigma=2, \nu^{\delta}=1, \varsigma_{1}=1$ and $\varsigma_{2}=1$.

Defining the fractal special function ${ }^{1}$

$$
\csc h_{\delta}\left(\psi^{\delta}\right)=\frac{2}{\mathrm{O}_{\delta}\left(\psi^{\delta}\right)-\mathrm{O}_{\delta}\left(-\psi^{\delta}\right)},
$$

we have

$$
\chi_{\delta}(\psi)=\beta_{1} \csc h_{\delta}^{2}\left(\beta_{2} \psi^{\delta}\right)
$$

such that

$$
\left(\frac{d^{\delta} \chi_{\delta}(\psi)}{d \psi^{\delta}}\right)^{2}+4 \beta_{2}^{2} \chi_{\delta}^{2}(\psi)+\frac{4 \beta_{2}^{2}}{\beta_{1}} \chi_{\delta}^{3}(\psi)=0 .
$$

Taking $\Theta_{\delta}(\psi)=\chi_{\delta}(\psi)$, we have from Eqs. (46) and (50) that

$$
4 \beta_{2}^{2}=\frac{\varsigma_{3}}{\varsigma_{2}}
$$

and

$$
\frac{4 \beta_{2}^{2}}{\beta_{1}}=\frac{\varsigma_{1}}{3 \varsigma_{2}} .
$$

Thus, we deduce from Eqs. (51) and (52) that

$$
\begin{aligned}
& \beta_{2}=\frac{\sqrt{\frac{\varsigma_{3}}{\varsigma_{2}}}}{2}, \\
& \beta_{1}=\frac{3 \varsigma_{3}}{\varsigma_{1}} .
\end{aligned}
$$

The non-differentiable solution of Eq. (43) is as follows:

$$
\Theta_{\delta}(\psi)=\frac{3 \varsigma_{3}}{\varsigma_{1}} \csc h_{\delta}^{2}\left(\frac{\sqrt{\frac{\varsigma_{3}}{\varsigma_{2}}}}{2} \psi^{\delta}\right) .
$$

We derive the exact traveling-wave solution for Eqs. (28) and (55) that

$$
\begin{aligned}
M_{\delta}(\mu, \tau)= & \frac{3\left(\sigma-\nu^{\delta}\right)}{\varsigma_{1}} \csc h_{\delta}^{2} \\
& \times\left[\frac{\sqrt{\frac{\sigma-\nu^{\delta}}{\varsigma_{2}}}}{2}\left(\mu^{\vartheta}-\nu^{\vartheta} \tau^{\vartheta}\right)\right] .
\end{aligned}
$$

Plot of Eq. (56) for the parameters $\sigma=2, \nu^{\delta}=1$, $\varsigma_{1}=1$ and $\varsigma_{2}=1$ (in Cantor condition $\delta=\ln 2 / \ln 3$ ) is illustrated in Fig. 1.

\section{CONCLUSION}

Based on the theory of LFD, the two- and threedimensional local fractional Boussinesq equations for the wave content in fractal domain were proposed. The non-differentiable-type traveling-wave transform is used to generalize the problem to the nonlinear local fractional ODE. Furthermore, the exact traveling-wave solution for the proposed model is also discussed. The proposed formulation is efficient for obtaining the exact traveling-wave solutions of the nonlinear local fractional PDEs.

\section{ACKNOWLEDGMENTS}

This work is supported by the State Key Research Development Program of the People's Republic of China (Grant No. 2016YFC0600705), the Natural 
Science Foundation of China (Grant No. 51323004), and the Priority Academic Program Development of Jiangsu Higher Education Institutions (PAPD2014).

\section{REFERENCES}

1. X. J. Yang, D. Baleanu and H. M. Srivastava, Local Fractional Integral Transforms and Their Applications (Academic Press, New York, 2015).

2. S. Liu, Z. Pan, W. Fu and X. Cheng, Fractal generation method based on asymptote family of generalized Mandelbrot set and its application, J. Nonlinear Sci. Appl. 10(3) (2017) 1148-1161.

3. S. Liu, W. Fu, L. He, J. Zhou and M. Ma, Distribution of primary additional errors in fractal encoding method, Multimed. Tools Appl. 76(4) (2017) 57875802.

4. W. Fu, J. Zhou, S. Liu, M. Ma and Y. Ma, Differential trajectory tracking with automatic learning of background reconstruction, Multimed. Tools Appl. 75(21) (2016) 13001-13013.

5. S. Liu, Z. Zhang, L. Qi and M. Ma, A fractal image encoding method based on statistical loss used in agricultural image compression, Multimed. Tools Appl. 75(23) (2016) 15525-15536.

6. V. E. Tarasov, Map of discrete system into continuous, J. Math. Phys. 47(9) (2006) 092901.

7. V. E. Tarasov, Partial fractional derivatives of Riesz type and nonlinear fractional differential equations, Nonlinear Dyn. 86(3) (2016) 1745-1759.

8. V. E. Tarasov, Fractional Dynamics: Application of Fractional Calculus to Dynamics of Particles, Fields and Media (Springer, New York, 2010).

9. B. J. West, M. Bologna and P. Grigolini, Physics of Fractal Operators (Springer, New York, 2012).

10. V. E. Tarasov, Fractional hydrodynamic equations for fractal media, Ann. Phys. 318(2) (2005) 286307.

11. E. Baskin and A. Iomin, Electrostatics in fractal geometry: Fractional calculus approach, Chaos Solitons Fractals 44(4) (2011) 335-41.

12. V. E. Tarasov, Fractional Fokker-Planck equation for fractal media, Chaos 15(2) (2005) 023102.

13. A. Carpinteri and P. Cornetti, A fractional calculus approach to the description of stress and strain localization in fractal media, Chaos Solitons Fractals 13(1) (2002) 85-94.

14. X. J. Yang, J. T. Machado, D. Baleanu and C. Cattani, On exact traveling-wave solutions for local fractional Korteweg-de Vries equation, Chaos 26(8) (2016) 084312.

15. X. J. Yang, J. T. Machado, C. Cattani and F. Gao, On a fractal LC-electric circuit modeled by local fractional calculus, Commun. Nonlinear Sci. Numer. Simul. 47 (2017) 200-206.
16. X. J. Yang, F. Gao and H. M. Srivastava, Exact travelling wave solutions for the local fractional twodimensional Burgers-type equations, Comput. Math. Appl. 73(2) (2017) 302-201.

17. H. Jafari, H. Tajadodi and S. J. Johnston, A decomposition method for solving diffusion equations via local fractional time derivative, Therm. Sci. 19 (2015) S123-S129.

18. D. Kumar, J. Singh and D. Baleanu, A hybrid computational approach for Klein-Gordon equations on Cantor sets, Nonlinear Dyn. 87(1) (2017) 511517.

19. S. S. Ye, S. T. Mohyud-Din and F. B. M. Belgacem, The Laplace series solution for local fractional Korteweg-de Vries equation, Therm. Sci. 20(3) (2016) S867-S870.

20. H. Y. Liu, J. H. He and Z. B. Li, Fractional calculus for nanoscale flow and heat transfer, Int. J. Numer. Meth. Heat Fluid Flow 24(6) (2014) 1227-1250.

21. H. Jafari, H. K. Jassi, F. Tchier and D. Baleanu, On the approximate solutions of local fractional differential equations with local fractional operators, Entropy 18(4) (2016) 150.

22. H. Budak, M. Z. Sarikaya and E. Set, Generalized Ostrowski type inequalities for functions whose local fractional derivatives are generalized s-convex in the second sense, J. Appl. Math. Comput. Mech. 15(4) (2016) 11-21.

23. M. Sarikaya and H. Budak, Generalized Ostrowski type inequalities for local fractional integrals, Proc. Am. Math. Soc. 145 (2017) 1527-1538.

24. K. M. Kolwankar and A. D. Gangal, Local fractional Fokker-Planck equation, Phys. Rev. Lett. 80(2) (1998) 214.

25. Y. Chen, Y. Yan and K. Zhang, On the local fractional derivative, J. Math. Anal. Appl. 362(1) (2010) 17-33.

26. A. Babakhani and V. Daftardar-Gejji, On calculus of local fractional derivatives, J. Math. Anal. Appl. 270(1) (2002) 66-79.

27. K. M. Kolwankar and A. D. Gangal, Hölder exponents of irregular signals and local fractional derivatives, Pramana 48(1) (1997) 49-68.

28. F. B. Adda and J. Cresson, About non-differentiable functions, J. Math. Anal. Appl. 263(2) (2001) 721737.

29. A. Carpinteri, P. Cornetti and K. M. Kolwankar, Calculation of the tensile and flexural strength of disordered materials using fractional calculus, Chaos Solitons Fractals 21(3) (2004) 623632.

30. C. S. Liu, On the local fractional derivative of everywhere non-differentiable continuous functions on intervals, Commun. Nonlinear Sci. Numer. Simul. 42 (2017) 229-235. 